\title{
Speeding-up the Fitting of the Model Defining the Ribs-bounded Contour
}

\author{
Mykolas J. Bilinskas ${ }^{1}$, Gintautas Dzemyda ${ }^{2}$, Martynas Sabaliauskas ${ }^{3}$ \\ ${ }^{1-3}$ Institute of Mathematics and Informatics, Vilnius University, Lithuania
}

\begin{abstract}
The method for analysing transversal plane images from computer tomography scans is considered in the paper. This method allows not only approximating ribs-bounded contour but also evaluating patient rotation around the vertical axis during a scan. In this method, a mathematical model describing the ribs-bounded contour was created and the problem of approximation has been solved by finding the optimal parameters of the mathematical model using least-squares-type objective function. The local search has been per-formed using local descent by quasi-Newton methods. The benefits of analytical derivatives of the function are disclosed in the paper.
\end{abstract}

Keywords - Analytical models, computed tomography, least squares approximation, medical diagnostic imaging.

\section{INTRODUCTION}

Computed tomography (CT) is a technology allowing the inside of objects to be spatially viewed using computerprocessed X-rays. It is very important in medical diagnostics because it shows human internal organs without cutting, e.g., brain, liver [1], and prostate [2]. CT scans are 3D images $-\mathrm{a}$ collection of 2D images (slices), representing slices by transversal plane. Many papers deal with the finding of the ribs-bounded contour [3], [4], [5]. The ribs-bounded contour restricts the region of location of internals in the slice. Internal organ localisation is particularly important for the comparison of patient health state or evaluation of disease processes. Here the registration problem of slices from different scans of the same patient becomes significant. The ribs-bounded contour may serve as a reference point in comparing two slices. Such comparison of slices should play an essential role in the registration of CT images. References that deal with the registration on images find a wide application in medicine [6], [7], [8], [9].

In the papers [3], [4], [5] a mathematical model is suggested to approximate the ribs-bounded contour and to evaluate the parameters of this model from the CT image. The local optimisation problem of some non-linear objective function is formulated. It is solved using quasi-Newton descent method. The experiments indicate that finding the optimal parameter values of this model is computationally expensive.

In the paper, we find the analytical derivatives of the objective function seeking to speed up the process of optimisation.

\section{DATA TO BE ANALYSED}

We investigate images of size $512 \times 512$ gathered by GE LightSpeed Pro 32 CT scanner ${ }^{1}$. 16-bit DICOM grayscale images were obtained using the scanner. The images were automatically linearly normalised to the interval $[0 ; 255]$ by window level (center) 40 and window width $350 \mathrm{HU}$ (Hounsfield units). The CT scanner uses a set of software algorithms to determine the amount of $\mathrm{x}$-radiation absorbed by every element in a plane of tissue. Each of these elements is represented by a pixel on the video display, and the density (amount of X-radiation absorbed) is measured in Hounsfield units (HU). Figure 1 demonstrates the possible content of particular scan slices. Depending on the slice, the heart, lungs, stomach, or liver, can be seen. In all the slices of Fig. 1, the internal organs are bounded by ribs. Figure 1 was obtained after the patient was given a radiocontrast agent injection. Therefore, the heart and aorta as well as all blood vessels are bright here.

\section{METHOD}

In this section, a method of analysis of CT scan slice (through transversal plane) is presented. This method allows us not only to find the ribs-bounded contour but also to evaluate patient rotation around the vertical axis during the scan.

We notice a symmetry of the ribs by the human sagittal plane, body rotation in respect to bed, aorta near spine, high grayscale intensity of bones, bed, heart and blood vessels (if the radiocontrast agent was injected to the patient). All these aspects should be considered when building a method.

The method consists of two steps [3]: (a) extracting the bone tissue from image; (b) approximating the ribs-bounded contour with a mathematical function.

\section{A. Ribs-bounded Contour Approximation}

As a result of the bone tissue extraction presented in [3], we get binary images - see the example in Fig. 2. Denote $B=\left\{\left(b_{1 i}, b_{2 i}\right), i=\overline{1, m}\right\}$ the set of coordinates of bone pixels obtained during analysis of CT image slices, $m$ is the number of bone pixels.

The ribs form a shape similar to cardioid (see Fig. 1):

$$
\rho=1+\cos (\varphi-\pi / 2), \varphi \in[-\pi / 2 ; 3 \pi / 2)
$$

${ }^{1}$ http://www3.gehealthcare.com/en/products/categories/goldseal_refurbished systems/goldseal computed tomography/goldseal vct series 
Here $\rho$ is the radius and $\varphi$ is the polar angle. The shape of (1) is depicted in Fig. 3 (thick curve). It looks similar because it features a cave, which could be used to approximate ribs cave near spine. $\pi / 2$ is introduced in (1) because the standard cardioid is rotated by $90^{\circ}$ as compared in Fig. 3 and the ribsbounded contour in the images should be oriented like ribs depicted in Fig. 1.

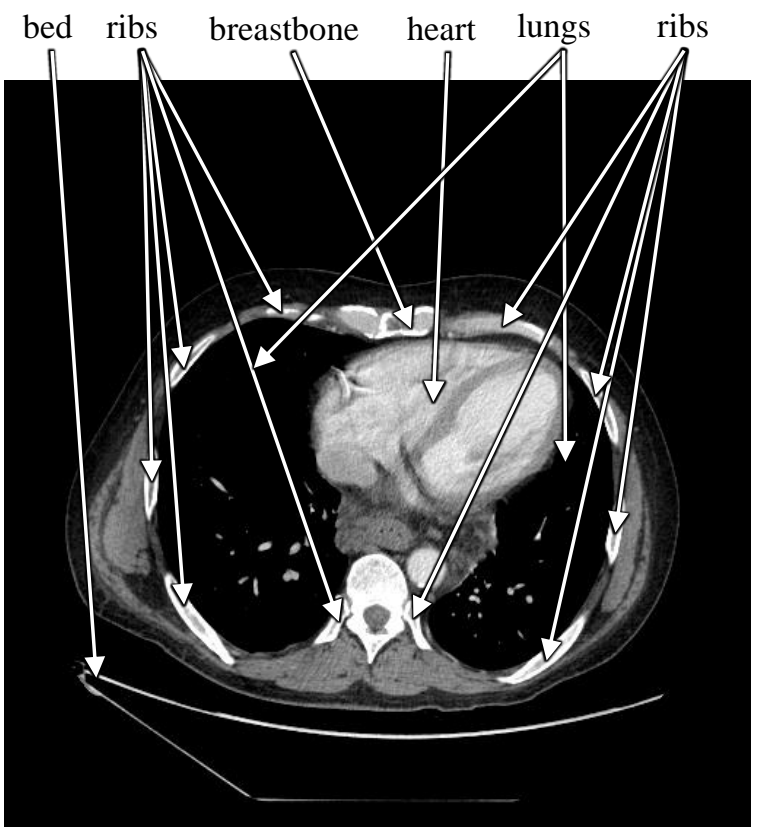

a)

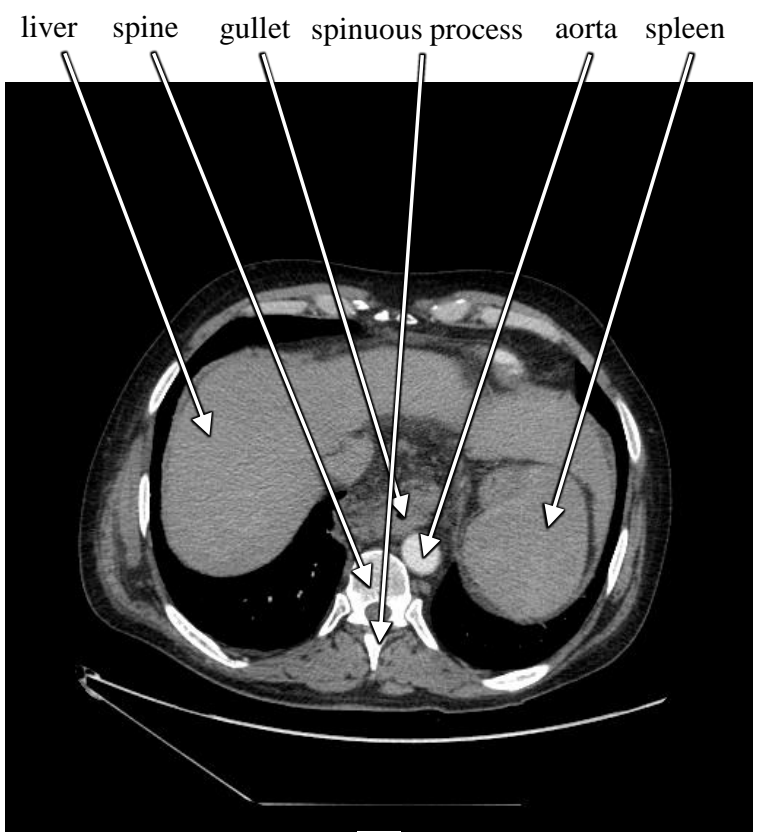

b)

Fig. 1. Examples of the CT image slices.

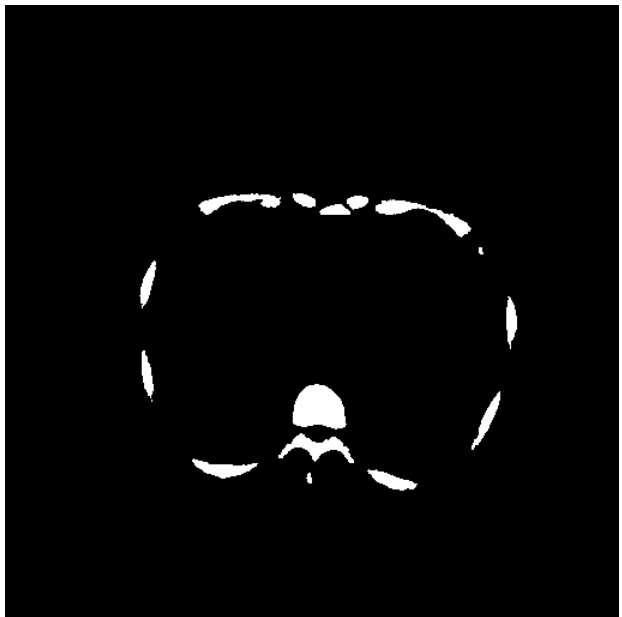

Fig. 2. Pixels representing bone tissue.

Our research showed that a ribs-bounded contour was more condensed vertically than the standard cardioid curve. Therefore, we suggest adding a new optimisable parameter power $s$ :

$$
\rho=(1+\cos (\varphi-\pi / 2))^{s}
$$

The (2) curve with different $s$ is depicted in Fig. 3.

As we see in Fig. 1, the rib-bounded contour has some rotation in respect to the bed. Therefore, we should introduce the angle $\theta$ of such rotation.

Parameter $s$ influences not only the vertical scale of the curve (1), but the form of the curve, too (see Fig. 3 for curves with different values of $s$ ).

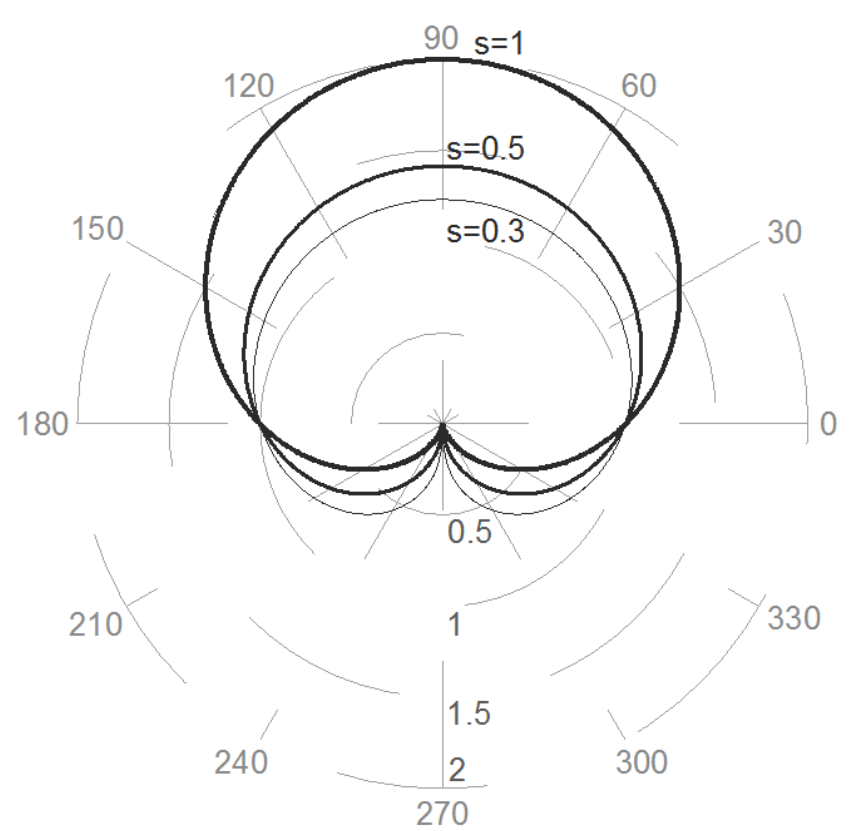

Fig. 3. Thick line - standard cardioid (1), middle thickness and thin lines - (2) curve with $s=0.5$ and $s=0.3$, respectively. 
In the CT scan slice (Fig. 1), we see a cave influenced by the breastbone. Curve (2) is convex in this region. Therefore, we need to complement the model (2) redefining $\rho$ with additional member $\rho^{\prime}$ whose form may vary depending on the cave:

$$
\rho=(1+\cos (\varphi-\pi / 2))^{s}-\rho^{\prime} .
$$

This member realises the cave by subtraction of some value from the right side of (2) starting from $\varphi=\pi / 2-\beta$ till $\varphi=\pi / 2+\beta \cdot \rho^{\prime}$ depends on $\varphi$ and has special properties. It should

a) be unimodal non-negative function on $\varphi$;

b) achieve the maximal value as $\varphi=\pi / 2$;

c) be symmetrical function in respect of $\varphi=\pi / 2$;

d) be equal to 0 when $\varphi=\pi / 2-\beta$ and $\varphi=\pi / 2+\beta$;

e) have zero first and second derivatives on $\varphi$ when $\varphi=\pi / 2-\beta$ and $\varphi=\pi / 2+\beta$.

Function $\rho^{\prime}$ may be as follows:

$$
\rho^{\prime}= \begin{cases}c \sin ^{l}(\pi(\varphi-\pi / 2+\beta) / 2 \beta) & \\ & \text { if } \beta \geq|\varphi-\pi / 2| \\ 0, & \text { else }\end{cases}
$$

In (4), we have three control parameters for which optimal values need to be found: $\beta$ is an angle defining the region of subtraction, $c$ defines the maximal value of subtraction, $l$ defines the steepness of curve describing the cave $(l \geq 2)$. See [3] for examples of $\rho^{\prime}$ depending on different values of $\beta, c, l$. Moreover, we need some additional parameters $a$ and $b$ that define the horizontal and vertical scales of the curve that approximate the rib-bounded contour, respectively.

The curve (3) should be fitted among ribs in the picture of bone tissue. For this reason, we need the optimal place of the point of (3) corresponding to $\rho=0$ in the picture; denote coordinates of this point by $\left(x_{0}, y_{0}\right)$.

If the values of $s, \theta, a, b, x_{0}, y_{0}, \beta, c, l$ are fixed, we can draw some parametric curve $(x, y)=(x(\varphi), y(\varphi))$ approximating the rib-bounded contour:

$$
\begin{aligned}
& x(\varphi)=x_{0}+a \rho(\varphi) \cos \varphi \cos \theta-b \rho(\varphi) \sin \varphi \sin \theta \\
& y(\varphi)=y_{0}+a \rho(\varphi) \cos \varphi \sin \theta+b \rho(\varphi) \sin \varphi \cos \theta
\end{aligned}
$$

where $\rho$ is defined by (3). If $\varphi$ runs through the interval $[-\pi / 2 ; 3 \pi / 2)$ with a step $2 \pi / n$, we get from (5) and (6) a sequence of points $C_{j}=\left(x_{j}, y_{j}\right), j=\overline{1, n}$ of the curve. In our experiments, $n=180$.

\section{B. Optimization Problem}

The model of ribs-bounded contour has nine parameters whose values can be varied seeking to find the best approximation of the contour: $s, \theta, a, b, x_{0}, y_{0}, \beta, c, l$. The optimal values of these parameters must be defined by the set $B$ of coordinates of bone pixels obtained during the analysis of CT image slices.

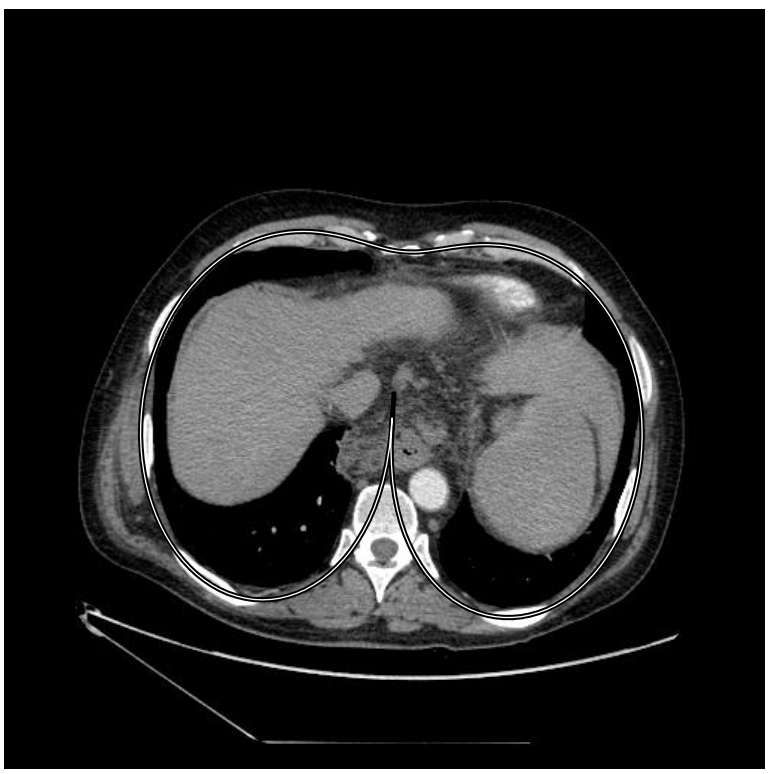

Fig. 4. The ribs-bounded contour.

The optimisation problem to find optimal $s, \theta, a, b, x_{0}, y_{0}$, $\beta, c, l$ is formulated as a least square one:

$$
\begin{aligned}
& \min _{s, \theta, a, b, x_{0}, y_{0}, \beta, c, l} f\left(s, \theta, a, b, x_{0}, y_{0}, \beta, c, l\right) \\
& f(\cdot)=\sum_{i}^{m}\left\|B_{i}-C_{k_{i}}\right\|^{2}, k_{i}=\arg \min _{j}\left\|B_{i}-C_{j}\right\|
\end{aligned}
$$

Here $k_{i}$ is the order number of the nearest model curve point among $n$ to the $i$ th bone tissue point.

The objective function is quite difficult because of the large number $m$ of bone tissue points. The optimisation method to solve (6) may be any local minimisation one. We use the Matlab realisation of the quasi-Newton method [10].

An example of optimisation and model curve fitting to the bone tissue is given in Figure 4 (the approximating curve is in black-white-black line).

Model (3)-(5) is suggested in [3]. More sophisticated model may be found in [4], [5]. The analytical derivatives were found especially for this model in [3].

\section{ANALYTICAL DERIVATIVES OF THE OBJECTIVE FUNCTION}

Experiments in [3], [4], [5] indicate the necessity for large computing expenses for optimisation of the objective function (6). The way to speed up the optimisation is using the analytical derivatives of the objective function. In this case, we save a large number of calculations of the objective function.

The Maple software ${ }^{2}$ was used to determine the derivatives of such complex objective function on $s, \theta, a, b, x_{0}, y_{0}, \beta$, $c, l$.

The derivatives are presented in (7)-(15):

\footnotetext{
${ }^{2}$ http://www.maplesoft.com
} 


$$
\begin{gathered}
\frac{d f}{d s}=\sum_{i}^{m}-2\left(1+\sin \left(\frac{2 \pi}{n} k_{i}\right)\right)^{s} \ln \left(1+\sin \left(\frac{2 \pi}{n} k_{i}\right)\right)\left(\left(b_{1 i}-\right.\right. \\
\left.-x_{k_{i}}\right)\left(a \cos \left(\frac{2 \pi}{n} k_{i}\right) \cos \theta+b \sin \left(\frac{2 \pi}{n} k_{i}\right) \sin \theta\right)+ \\
\left.+\left(b_{2 i}-y_{k_{i}}\right)\left(a \cos \left(\frac{2 \pi}{n} k_{i}\right) \sin \theta+b \sin \left(\frac{2 \pi}{n} k_{i}\right) \cos \theta\right)\right) \\
\frac{d f}{d \theta}=\sum_{i}^{m} 2\left(b_{1 i}-x_{k_{i}}\right)\left(a \rho\left(\frac{2 \pi}{n} k_{i}\right) \cos \left(\frac{2 \pi}{n} k_{i}\right) \cos \theta+\right. \\
\left.+b \rho\left(\frac{2 \pi}{n} k_{i}\right) \sin \left(\frac{2 \pi}{n} k_{i}\right) \sin \theta\right)+ \\
2\left(b_{2 i}-\quad y_{k_{i}}\right)\left(-a \rho\left(\frac{2 \pi}{n} k_{i}\right) \cos \left(\frac{2 \pi}{n} k_{i}\right) \cos \theta+\right. \\
\left.+b \rho\left(\frac{2 \pi}{n} k_{i}\right) \sin \left(\frac{2 \pi}{n} k_{i}\right) \sin \theta\right) \\
\frac{d f}{d a}=\sum_{i}^{m}-2 \rho\left(\frac{2 \pi}{n} k_{i}\right) \cos \left(\frac{2 \pi}{n} k_{i}\right)\left(\left(b_{1 i}-x_{k_{i}}\right) \cos \theta+\right. \\
\left.+\left(b_{2 i}-y_{k_{i}}\right) \sin \theta\right) \\
\frac{d f}{d b}=\sum_{i}^{m} 2 \rho\left(\frac{2 \pi}{n} k_{i}\right) \sin \left(\frac{2 \pi}{n} k_{i}\right)\left(\left(b_{1 i}-x_{k_{i}}\right) \sin \theta-\right. \\
\left.-\left(b_{2 i}-y_{k_{i}}\right) \cos \theta\right) \\
\frac{d f}{d x_{0}}=\sum_{i}^{m}-2\left(b_{1 i}-x_{k_{i}}\right) \\
\frac{d f}{d y_{0}}=\sum_{i}^{m}-2\left(b_{2 i}-y_{k_{i}}\right)
\end{gathered}
$$

$\frac{d f}{d \beta}=\sum_{i}^{m} 2 c \sin ^{1-1}\left(\frac{\pi}{2 \beta}\left(\frac{2 \pi}{n} k_{i}-\frac{\pi}{2}+\beta\right)\right) l \cos \left(\frac{\pi}{2 \beta}\left(\frac{2 \pi}{n} k_{i}-\frac{\pi}{2}+\right.\right.$ $+\beta)) \frac{\pi}{2 \beta^{2}}\left(\frac{\pi}{2}-\frac{2 \pi}{n} k_{i}\right)\left(\left(b_{1 i}-x_{k_{i}}\right)\left(a \cos \left(\frac{2 \pi}{n} k_{i}\right) \cos \theta-\right.\right.$ $\left.-b \sin \left(\frac{2 \pi}{n} k_{i}\right) \sin \theta\right)+\left(b_{2 i}-y_{k_{i}}\right)\left(a \cos \left(\frac{2 \pi}{n} k_{i}\right) \sin \theta-\right.$$$
\left.\left.-b \sin \left(\frac{2 \pi}{n} k_{i}\right) \cos \theta\right)\right)
$$

$$
\frac{d f}{d c}=
$$

$\sum_{i}^{m} 2 \sin ^{l}\left(\pi\left(\frac{2 \pi}{n} k_{i}-\frac{\pi}{2}+\beta\right) / 2 \beta\right)\left(\left(b_{1 i}-\right.\right.$

$$
\begin{gathered}
\left.-x_{k_{i}}\right)\left(a \cos \left(\frac{2 \pi}{n} k_{i}\right) \cos \theta+b \sin \left(\frac{2 \pi}{n} k_{i}\right) \sin \theta\right)+ \\
\left.+\left(b_{2 i}-y_{k_{i}}\right)\left(a \cos \left(\frac{2 \pi}{n} k_{i}\right) \sin \theta+b \sin \left(\frac{2 \pi}{n} k_{i}\right) \cos \theta\right)\right)(14) \\
\frac{d f}{d l}=\sum_{i}^{m} 2 c \sin ^{l}\left(\frac{\pi\left(\frac{2 \pi}{n} k_{i}-\frac{\pi}{2}+\beta\right)}{2 \beta}\right) \ln \left(\sin \left(\frac{\pi\left(\frac{2 \pi}{n} k_{i}-\frac{\pi}{2}+\beta\right)}{2 \beta}\right)\right)\left(\left(b_{1 i}-\right.\right. \\
\left.-x_{k_{i}}\right)\left(a \cos \left(\frac{2 \pi}{n} k_{i}\right) \cos \theta+b \sin \left(\frac{2 \pi}{n} k_{i}\right) \sin \theta\right)+ \\
+\left(b_{2 i}-y_{k_{i}}\right)\left(a \cos \left(\frac{2 \pi}{n} k_{i}\right) \sin \theta+\right. \\
\left.\left.+b \sin \left(\frac{2 \pi}{n} k_{i}\right) \cos \theta\right)\right)
\end{gathered}
$$

Here $\left(b_{1 i}, b_{2 i}\right), i=\overline{1, m}$ are the coordinates of bone pixels obtained during analysis of CT image slices, $m$ is the number of bone pixels.

The experiments in [4], [5] have shown that optimal values of parameter $\beta$ are close to $\pi$. Therefore, $\beta$ can be fixed at $\pi$, i.e. it may be set as non-optimisable parameter. This means that the subtrahend for breastbone is subtracted from $\rho(\varphi)$ for all $\varphi \in[-\pi / 2 ; 3 \pi / 2]$, and the shape of the breastbone cave is controlled by scale $c$ and power $l$ only. The model (3) in polar coordinates becomes

$$
\rho(\varphi)=\left(1+\cos \left(\varphi-\frac{\pi}{2}\right)\right)^{s}-c \sin ^{l}\left(\left(\varphi+\frac{\pi}{2}\right) / 2\right) .
$$

The derivatives of the objective function $f(\cdot)$ become simpler. Most derivatives remain as they stand before, derivative (13) is not applied, and derivatives (14) and (15) become (17) and (18), respectively:

$$
\begin{gathered}
\frac{d f}{d c}=\sum_{i}^{m} 2 \sin ^{l}\left(\frac{\pi}{n} k_{i}+\frac{\pi}{4}\right)\left(( b _ { 1 i } - x _ { k _ { i } } ) \left(a \cos \left(\frac{2 \pi}{n} k_{i}\right) \cos \theta+\right.\right. \\
\left.+b \sin \left(\frac{2 \pi}{n} k_{i}\right) \sin \theta\right)+\left(b_{2 i}-y_{k_{i}}\right)\left(a \cos \left(\frac{2 \pi}{n} k_{i}\right) \sin \theta+\right. \\
\left.\left.+b \sin \left(\frac{2 \pi}{n} k_{i}\right) \cos \theta\right)\right)(17) \\
\frac{d f}{d l}=\sum_{i}^{m} 2 c \sin ^{l}\left(\frac{\pi}{n} k_{i}+\frac{\pi}{4}\right) \ln \left(\sin \left(\frac{\pi}{n} k_{i}+\frac{\pi}{4}\right)\right)\left(\left(b_{1 i}-\right.\right. \\
\left.-x_{k_{i}}\right)\left(a \cos \left(\frac{2 \pi}{n} k_{i}\right) \cos \theta+b \sin \left(\frac{2 \pi}{n} k_{i}\right) \sin \theta\right)+ \\
+\left(b_{2 i}-y_{k_{i}}\right)\left(a \cos \left(\frac{2 \pi}{n} k_{i}\right) \sin \theta+\right. \\
\left.\left.+b \sin \left(\frac{2 \pi}{n} k_{i}\right) \cos \theta\right)\right) .(18
\end{gathered}
$$

\section{V.CONCLUSION}

In the paper, a method for analysing transversal plane images from computer tomography scans has been analysed. This method allows not only approximating ribs-bounded contour but also evaluating patient rotation around the vertical axis during a scan. In this method, a mathematical model describing the ribs-bounded contour has been created and the problem of approximation has been solved by finding out the optimal parameters of the mathematical model using leastsquares-type objective function. The local search has been performed using local descent by quasi-Newton methods. The proposed approximation defines the ribs-bounded contour exactly. The model may be applied to any $2 \mathrm{D}$ slice where the ribs are visible.

The experiments show that application of analytical derivatives of the objective function allows speeding up the optimisation by 1.98 times. This is essential benefit when the medical doctors need fast decisions.

\section{REFERENCES}

[1] H. Nugroho, D. Ihtatho, and H. Nugroho, "Contrast enhancement for liver tumor identification," The MIDAS Journal - Grand Challenge Liver Tumor Segmentation (2008 MICCAI Workshop), 2008.

[2] S. Chen, D. M. Lovelock, and R. J. Radke, "Segmenting the prostate and rectum in CT imagery using anatomical constraints," Medical Image Analysis, vol. 15, no. 1, pp. 1-11, Feb. 2011. https://doi.org/10.1016/j.media.2010.06.004

[3] M. J. Bilinskas, G. Dzemyda, and M. Trakymas, "Computed tomography image analysis: The model of ribs-bounded contour," vol. 2503. Plzen, Czech Republic: Vaclav Skala - UNION Agency, 2015, pp. 81-84.

[4] M. J. Bilinskas and G. Dzemyda, "Optimization in modeling the ribsbounded contour from computer tomography scan," in Numerical computations: theory and algorithms (NUMTA-2016): proceedings of 
the 2nd international conference, vol. 1776. Pizzo Calabro, Italy: AIP Publishing, 2016. https://doi.org/10.1063/1.4965405

[5] M. J. Bilinskas and G. Dzemyda, "Modelling the ribs-bounded contour in computer tomography images," in Meždunarodnyj kongress po informatike: Informacionnye sistemy $i$ tehnologii : Materialy meždunarodnogo naučnogo kongressa Respublika Belarus. Minsk, Belarus: BGU, 2016, pp. 198-203. [Online]. Available: http://elib.bsu.by/bitstream/123456789/159801/1/Dzemyda_Bilinskas.pdf

[6] P. Treigys, V. Šaltenis, G. Dzemyda, V. Barzdžiukas, and A. Paunksnis, "Automated optic nerve disc parameterization," Informatica, vol. 19, no. 3, pp. 403-420, 2008.

[7] F. Graf, H.-P. Kriegel, M. Schubert, S. Pölsterl, and A. Cavallaro, "2D image registration in CT images using radial image descriptors," in Medical Image Computing and Computer-Assisted Intervention MICCAI 2011: 14th International Conference, Toronto, Canada, September 18-22, 2011, Proceedings, Part II, G. Fichtinger, A. Martel, and T. Peters, Eds. Berlin, Heidelberg: Springer Berlin Heidelberg, 2011, pp. 607-614. https://doi.org/10.1007/978-3-642-23629-7 74

[8] F. P. M. Oliveira and J. M. R. S. Tavares, "Registration of plantar pressure images," International Journal for Numerical Methods in Biomedical Engineering, vol.28, no. 6-7, pp. 589-603, Aug. 2011. https://doi.org/10.1002/cnm.1461

[9] Á. Fernández, N. Rabin, R. R. Coifman, and J. Eckstein, "Diffusion methods for aligning medical datasets: Location prediction in CT scan images," Medical Image Analysis, vol. 18, no. 2, pp. 425-432, Feb. 2014. https://doi.org/10.1016/j.media.2013.12.009

[10] Mathworks, "Unconstrained Nonlinear Optimization Algorithms," 2016. [Online]. Available: http://se.mathworks.com/help/optim/ug/ unconstrained-nonlinear-optimization-algorithms.html

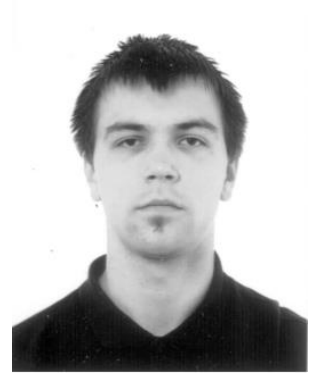

Mykolas J. Bilinskas received BA in software engineering in 2011 and MS in computer modelling in 2013 from Vilnius University, Faculty of Mathematics and Informatics. He obtained experience in software development at CERN (European Organisation for Nuclear Research) in Switzerland and at JSC Altechna. Currently he is a $\mathrm{PhD}$ student at Vilnius University, Institute of Mathematics and Informatics. His interests include computer vision and computational geometry.

E-mail: mykolas@bilinskas@mii.vu.lt

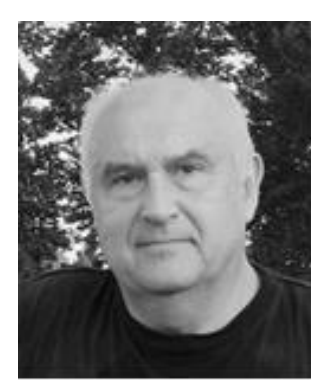

Gintautas Dzemyda received the Doctoral degree in technical sciences in 1984, and the degree of Doctor Habilius in 1997 from Kaunas University of Technology. He was conferred the title of Professor (1998) at Kaunas University of Technology. He is a full member of the Lithuanian Academy of Sciences (2011). Recent employment is at Vilnius University, Institute of Mathematics and Informatics as the Director of the Institute, a Head of Cognitive Computing Group and Principal Researcher.

The research interests cover visualisation of multidimensional data, optimisation theory and applications, data mining in databases, multiple criteria decision support, neural networks, parallel optimisation, image analysis. He is the author of more than 240 scientific publications, two monographs, five textbooks. He is the editor in chief of the international journals Informatica and Baltic Journal of Modern Computing. $\mathrm{He}$ is also the member of editorial boards of seven international journals.

E-mail: gintautas.dzemyda@mii.vu.lt

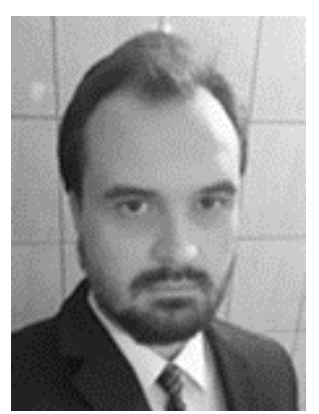

Martynas Sabaliauskas received BA (2010) and MS (2012) in mathematics from Vilnius University, Faculty of Mathematics and Informatics. He completed his Doctoral studies in informatics engineering in 2016 at Vilnius University, Institute of Mathematics and Informatics and developed his Doctoral Thesis for defending in 2017. His research experience includes designing, performing, and analysing of surface parameterisation and segmentation algorithms, sparse matrix processing algorithms, graph drawing algorithms, and game theory problems.

His research interests include game theory, mesh processing algorithms, parallel programming, and optimisation methods.

E-mail: Martynas.Sabaliauskas@mii.vu.lt 